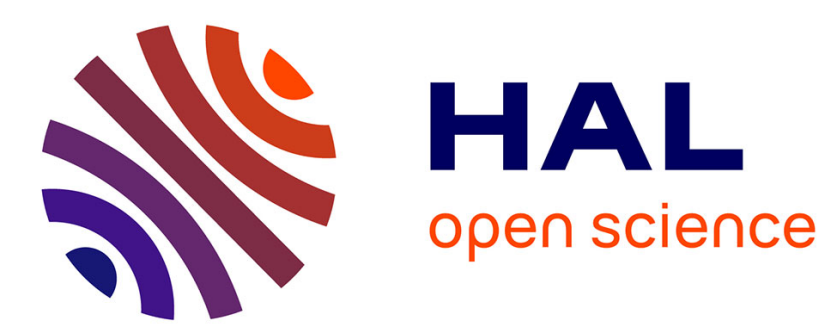

\title{
Delay electro-optic dynamics for brain inspired information processing
}

\author{
M. Jacquot, R. Martinenghi, S. Rybalko, Y.K. Chembo, L. Larger
}

\section{To cite this version:}

M. Jacquot, R. Martinenghi, S. Rybalko, Y.K. Chembo, L. Larger. Delay electro-optic dynamics for brain inspired information processing. Fifth 'Rio de la Plata' workshop on laser dynamics and nonlinear photonics, Dec 2011, Colonia del Sacramento, Uruguay. 10.1109/LDNP.2011.6162074 . hal-00661200

\section{HAL Id: hal-00661200 https://hal.science/hal-00661200}

Submitted on 18 Apr 2021

HAL is a multi-disciplinary open access archive for the deposit and dissemination of scientific research documents, whether they are published or not. The documents may come from teaching and research institutions in France or abroad, or from public or private research centers.
L'archive ouverte pluridisciplinaire HAL, est destinée au dépôt et à la diffusion de documents scientifiques de niveau recherche, publiés ou non, émanant des établissements d'enseignement et de recherche français ou étrangers, des laboratoires publics ou privés. 


\title{
Delay electro-optic dynamics for brain inspired information processing
}

\author{
M. Jacquot, R. Martinenghi, S. Rybalko, Y. Chembo, \\ L. Larger \\ UMR CNRS FEMTO-ST 6174, Optics dpt \\ University of Franche-Comté \\ Besançon, France \\ maxime.jacquot@univ-fcomte.fr
}

\begin{abstract}
This work reports on the first experimental photonic demonstration of a neuromorphic computational unit, on the basis of a recently proposed brain-inspired paradigm typically referred as Echo State Network, Liquid State Machine, or also Reservoir Computing in the neuronal computing literature. This paradigm makes use of the computational power offered by high dimensional transient motions developed by complex nonlinear dynamical systems, when the latter are excited by the information to be processed. The originality of the proposed photonic implementation is to exploit the dynamical complexity of delay dynamics, instead of that provided by spatially extended networks of dynamical nodes (as typically proposed in the existing literature). Complex delay dynamics are indeed well known in photonics with many different practical implementations. Our results have been obtained via a hybrid optoelectronic architecture, which has been successfully used in the past in the framework of optical chaos communications. We will report on two practical implementations involving whether wavelength or intensity dynamics subject to a single nonlinear delayed feedback, or even a multiple delayed one with randomly defined weights for each delay. The computational performance is successfully tested on a benchmark test, a spoken digit recognition task, with which state of the art performances are achieved.
\end{abstract}

Keywords : Nonlinear Delay Dynamics; photonic Reservoir Computing; information processing

\section{INTRODUCTION}

The brain research and neural network computing communities proposed independently in the early 2000 novel computational principles [1], which are suspected to mimic actual calculation and processing tasks that have been observed and studied in the brain. This computational principles referred as Echo State Network (ESN [2]), or Liquid State Machine (LSM, [3]), and also with the generic term Reservoir Computing ( $\mathrm{RC}$ ), is definitely different with respect to the standard Turing Machine principles widely implemented in electronic digital processor. Instead of processing the calculation tasks step by step with static states stored in memories, this new principle is based on computational power developed by complex nonlinear transient motions. These motions are typically developed in the high dimensional phase space of nonlinear dynamical systems, when the latter is excited by an input signal representing the information to be processed. The complex dynamics is usually materialized by a network of neurons (as in the brain), or by any spatially extended network of coupled nonlinear dynamical nodes. The corresponding generic architecture is depicted in Fig.1(a),
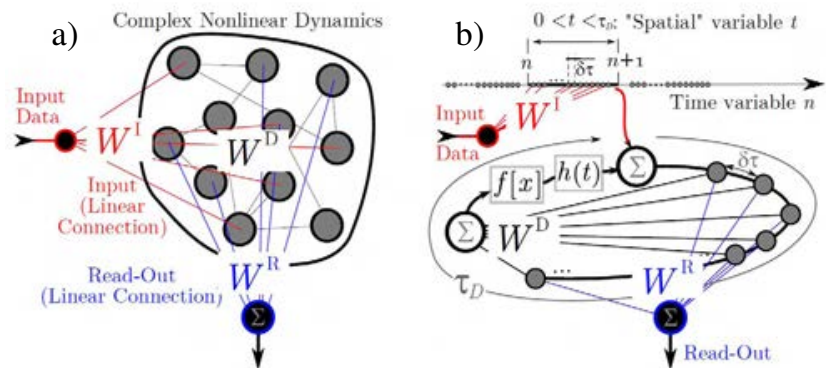

Figure 1. Principles of a NTC. a) : with a spatially extended dynamical network. b) : with a multiple delay feedback dynamics.

where strong similarities can be seen compared to standard recurrent neural network ( $\mathrm{RNN})$ : an input layer is dedicated to the injection of the input information (input connectivity matrix $\mathrm{W}^{\mathrm{I}}$ ) into a complex interconnected network of dynamical nodes (internal network connectivity matrix $\mathrm{W}^{\mathrm{D}}$ ); an output layer (Read Out matrix $\mathrm{W}^{\mathrm{R}}$ ) is dedicated to the extraction of the result, computed from the nonlinear transient developed by the network dynamics consequently to the injected input signal. The internal network connectivity matrix $\mathrm{W}^{\mathrm{D}}$ allows to perform the multiple delay dynamics, whereas the single delay ones are obtained for a connectivity matrix $\mathrm{W}^{\mathrm{D}}$ filled in with the same values of weights coefficients. Since one of our aim is to transpose these concepts into Physics and into a real world experimental demonstrator, our system will be referred in the remaining part of the paper, as NTC (Nonlinear Transient Computing [4]). NTC is suggested with the intention to reflect more clearly the actual physical origin of the approach, in a way which is expected to be more meaningful for physicists and for the nonlinear dynamics community, although historically the computer or brain science communities are referring to ESN, LSM or RC. 
One strong difference in NTC compared to RNN is the following: the internal network structure $\mathrm{W}^{\mathrm{D}}$ is assumed to be fixed (and not tunable according to a learning procedure as it is for RNN), and the learning procedure in NTC is aimed at determining the linear Read-Out $\mathrm{W}^{\mathrm{R}}$ only (which coefficients are typically optimized via a regression technique applied to a so-called learning set, consisting of known input-output couples for a given problem).

A significant innovation in NTC was recently proposed [5] by the nonlinear dynamics community, as a simple and efficient solution intended for real-world physical implementation, instead of the classical view of a network of nonlinear nodes [6, 7]. This novel approach can be justified by a known analogy between a delay dynamics and a spatiotemporal one (as a RNN). Both are infinite dimensional, and the space-time representation of a delay dynamics was already proposed 20 years ago [8], introducing on one hand a discrete time variable corresponding to one delay step forward, and on the other hand a virtual continuous space variable corresponding to the short time scale fluctuations within a time delay interval. As illustrated in Fig.1(b) virtual network nodes in a delay dynamics can be defined as positions within a time delay interval $\tau_{\mathrm{D}}$ separated by a "node distance" [5]. The connectivity of the resulting virtual spatio-temporal network is achieved via two dynamical mechanisms. The neighboring nodes are linearly coupled via the characteristic time $(\tau \approx 5 \delta \tau$ for an optimal adjacent node spacing, [5]) of the oscillator impulse response $\mathrm{h}(\mathrm{t})$. Each node is also nonlinearly coupled (nonlinear function $\mathrm{f}[\mathrm{x}]$ ) to its delayed feedback echo (total delay $\tau_{\mathrm{D}}$ ), and also to any additional delay eventually involved in the feedback. This is typically what is implemented in our demonstrator, where the kth node is fed back to the input of the nonlinear function, with a delay $\mathrm{k} \delta \tau(\mathrm{k}=1 \ldots \mathrm{N})$, and with a weight $w_{k}{ }_{k}$. Compared to a single delay dynamics [5], we explore the possibility to lower the number of nodes required for a comparable task (from 400 to 150 only), compensating this by an improved delay dynamics connectivity via a multiple delay topology with random weights (uniform distribution). In terms of computational efficiency, we reached with the multiple delay dynamics a word error rate (WER) comparable to single delay ones, for the same benchmark test consisting of a so-called "at any time" classification of spoken digits.

The operating principles for our NTC implemented with a delay feedback nonlinear dynamics can be summarized as follow (Fig.1(b)). An input information to be processed is exciting each virtual spatial node of the NTC dynamics; this addressing is performed by a kind of temporal division multiplexing, consisting in spreading each input sample over all the nodes within a time delay interval, according to an input connectivity matrix $\mathrm{W}^{\mathrm{I}}$. The NTC dynamics experiences, for each injected set of input information samples, a transient motion in its complex phase space. This motion is ruled by: (i) a nonlinear (function $\mathrm{f}[\mathrm{x}]$ ) delay feedback ( $\mathrm{N}$ delays $\mathrm{k} \delta \tau$ with weights $\mathrm{w}_{\mathrm{k}}^{\mathrm{D}}, \mathrm{k}=1$ to $\mathrm{N}$, and $\tau_{\mathrm{D}}=\mathrm{N} \delta \tau$, the coefficients $\mathrm{w}_{\mathrm{k}}{ }_{\mathrm{k}}$ are equal to 1 for a single delay topology); (ii) a linear impulse response $\mathrm{h}(\mathrm{t})$ limiting the rate of change of the feedback loop. The computed output is obtained by a Read-Out consisting of a linear combination of the amplitudes of each virtual node during the full transient; the Read-Out coefficients from $\mathrm{W}^{\mathrm{R}}$ are determined after a learning phase which corresponds to solving a regression problem from known pairs of input / response.

The next section will describe the experimental setup and the dynamical modeling of the NTC and its operating parameters conditions.

\section{NONLINEAR SINGLE OR MULTIPLE DELAY DYNAMICS}

The experimental setup is based on an original architecture intended to perform an Ikeda-like optoelectronic nonlinear delay dynamics [9-11] initially developed for secure chaos communications. We propose two practical implementations involving whether wavelength or intensity dynamics subject to a single nonlinear delayed feedback, or even a multiple delayed one with randomly defined weights for each delay.

The nonlinear function $\left(\mathrm{f}[\mathrm{x}]=\sin ^{2}(\mathrm{x})\right)$ is provided by tunable interference phenomena for both experiments: an imbalanced birefringent interferometer for the wavelength dynamics and an integrated telecom Mach-Zehnder modulator $\left(\mathrm{LiNbO}_{3}\right)$ for intensity dynamics. The nonlinear device output is detected by a photodiode, and an electronic feedback circuit performs a low pass or a bandpass filtering ruling the dynamics of the oscillator. This filtered signal serves as the Read-Out of the NTC. The input information is then added to the filtered signal, the resulting sum being multiple delayed (or single delayed) by an FPGA (Field Programmable Gate Array) board (wavelength dynamics) or by an optical fiber (intensity single delay dynamics). The advantage of the FPGA is that it serves here as a flexible and programmable delay line, in which several elementary FIFO memories (First In First Out) are implemented together with the weights $\mathrm{w}_{\mathrm{k}}^{\mathrm{D}}$ of each delayed feedback, as depicted in Fig.1(b). The weighted sum of the multiple delayed signals is amplified, combined with an offset, and serves finally as the input drive of the nonlinear device in order to close the loop of the oscillator.

The continuous time dynamics is ruled by a differential, or integro-differential, equation [12], thus defining one, or two, response time(s) associated to the low, or low and high, cutoff frequencies of the Fourier domain corresponding low pass, or bandpass, filter. Such a filter in the feedback loop dynamics, is driven by the nonlinear delayed term. The operating point of the dynamics along the nonlinear $\sin ^{2}$ - function is set by an offset phase added on the delayed feedback signal. The amplification feedback loop gain is referred as $\beta$. The delayed feedback coefficients $\mathrm{w}_{\mathrm{k}}^{\mathrm{D}}$ are programmed in the FPGA, they are defined randomly from a uniform distribution; compared to the single delay case, and they are introduced for an enhanced connectivity (in the framework of an equivalent complex network of dynamical nodes). In Fig. 1(b), $\delta \tau$ is an elementary time delay determining the spacing between two adjacent virtual nodes of the equivalent network of nodes approach for the complex dynamics. Depending on the chosen relative time scales, there are $N=150$ different nodes in the wavelength setup and $\mathrm{N}=400$ nodes in intensity setup. With the FPGA configuration for the delay, we are able to implement also as many as there are nodes.

Finally, in order to ensure a stable fixed point solution for an input free operation (autonomous dynamics), the feedback gain $\beta$ has to be set below the Hopf oscillation threshold 
defined as 1 . In the experiment $\beta$ is set to ca. 0.5 . The offset phase fixing the operating point of the NTC, is set to two characteristic position, $\pi / 4$ (parabolic non linearity, expected to be the optimal point), and $\pi / 2$ (around the linear operating point, which however becomes cubic in the wavelength dynamics since the amplitude span is larger than in the intensity dynamics). Thus, the amplitude of the input information can be set so that, in the wavelength dynamics, it induces a large scan of the nonlinear function (more than 2 extrema); which is a specific advantageous feature in comparison to the intensity dynamics.

When an input information signal has to be processed, it needs to be properly formatted relatively to the time scales and topology of the delay dynamics: each information sample has to maintain a transient state for each individual node of the delay dynamics. This is achieved by the introduction of an input connectivity matrix $\mathrm{W}^{\mathrm{I}}$, randomly, but uniquely defined for each input information signal. The temporal waveform to be injected in the setup input data port, is computer processed according to $\mathrm{W}^{\mathrm{I}}$ multiplied by a $2 \mathrm{D}$ representation of the input information signals (the digit cochleagram in the case of the spoken digit recognition task, see below). The corresponding 1D waveform is injected into the optoelectronic delay dynamics via an arbitrary waveform generator (AWG, Lecroy ArbStudio 1102). For each input information signal, the full transient response is recorded by a digital storage oscilloscope. An off-line post-processing is performed for both the training and testing stages. The training consists in the solving of a regression problem, and results in the definition of Read-Out coefficients of $\mathrm{W}^{\mathrm{R}}$ leading to an optimally correct answer on a training subset of input / answer pairs. The efficiency of such an optimal Read-Out is finally evaluated on a complementary subset of input / answer pairs. In principle, the FPGA could be also programmed to implement a direct on-line Read-Out, as soon as the coefficients are determined after the training. For sake of simplicity, this testing phase was fully processed offline, right after the training phase.

\section{SPOKEN DIGIT RECOGNITION TASK}

This task is a standard one already used in other RNN or NTC reports [5]. Even if not constituting a difficult one, it essentially allows for a relative evaluation of the computational efficiency and accuracy of our proposed NTC topology, moreover by comparison with other existing approaches..

The main goal of this standard classification test is to recognize a pronounced digit among the ten possible ones from 0 to 9 . The spoken digit data base used in this test corresponds to 500 digits extracted from the TI46 speech corpus. The digits are pronounced by 10 different female speakers uttering 5 times the 10 digits. Following a standard pre-processing task typically performed in many similar acoustic speech recognition task, the $1 \mathrm{D}$ acoustic waveform sampled at 12.5 $\mathrm{kHz}$ is transformed into a $2 \mathrm{D}$ frequency-time representation, a so-called cochleagram (the Lyon cochlear ear model). The performance for this task is characterized by the word error rate (WER), as well as a margin to the closest competitor digit. By tuning the experimental control parameters $(\beta$ and offset phase), state of the art performances have been obtained with a WER below $1 \%$.
Thus, we have demonstrated experimentally the efficiency of a photonic neuromorphic analog processor referred as NTC (nonlinear transient computer), which successfully passed a spoken digit recognition test with a WER similar to the state of the art. The reported results are obtained with an NTC based on a wavelength multiple / single or an intensity single delay nonlinear dynamics. In the wavelength nonlinear dynamics, the number of nodes is 3 times smaller than intensity single delay dynamics. NTC with delay dynamics is confirmed as an efficient solution for the practical implementation of this brain inspired computational paradigm, potentially providing experimental efficiency (in terms of speed) and flexibility (in terms of dynamics connectivity).

\section{PERSPECTIVES}

Future work will be devoted to the design of ultra-fast photonic versions making use of standard optical telecommunication devices and principles [11]. Many fundamental issues are also remaining, whether in order to quantify and to correlate the dynamical features of delay systems to the computational power, or to adapt the dynamics topology and the system architecture for the implementation of more advanced computational features (plasticity and integrated learning capability), thus allowing self-adaptation of the system to many different classes of complex problems. Work is in progress to address experimentally not only classification tasks, but also prediction ones on complex problems.

\section{ACKNOWLEDGMENT}

This work was mainly supported by the European PHOCUS project (FP7-2009-ICT-240763). We also acknowledge the additional support of the Institut universitaire de France.

\section{REFERENCES}

[1] H. Jaeger, W. Maass, and J. Principe, "Neural Networks" 20, 287 (2007).

[2] H. Jaeger and H. Haas, Science 304, 78 (2004).

[3] W. Maass, T. Natschläger, and H. Markram, Neural Comput. 14, 2531 (2002).

[4] N. Crook, Neurocomp. 70, 1167 (2007).

[5] L. Appeltant, M. C. Soriano, G. Van der Sande, J. Danckaert, S. Massar, J. Dambre, B. Schrauwen, C. R. Mirasso, and I. Fischer, Nature Commun. 2, 1 (2011).

[6] K. Vandoorne, W. Dierckx, B. Schrauwen, D. Verstraeten, R. Baets, P. Bienstman, and J. Campenhout, Opt. Expr. 16, 11182 (2008).

[7] K.T Vandoorne, J. Dambre, D. Verstraeten, B. Schrauwen, P. Bienstman, IEEE Transactions on Neural Networks, 22(9), 1469-1481 (2011)

[8] F. T. Arecchi, G. Giacomelli, A. Lapucci, and R. Meucci, Phys. Rev. A 45, R4225 (1992).

[9] J.-P. Goedgebuer, L. Larger, and H. Porte, Phys. Rev. Lett. 80, 2249 (1998).

[10] L. Larger and J. M. Dudley, Nature 465, 41 (2010).

[11] R. Lavrov, M. Jacquot, and L. Larger, IEEE J. Quantum Electron. 46, 1430 (2010).

[12] Y. Chembo Kouomou, P. Colet, L. Larger and N. Gastaud, Phys. Rev. $\begin{array}{lll}\text { Lett. } & \text { 95, } & 203903\end{array}$ 